\title{
Variation in hospital caesarean section rates for women with at least one previous caesarean section: a population based cohort study
}

\author{
Kathrin Schemann ${ }^{1,2^{*}}$, Jillian A. Patterson ${ }^{1}$, Tanya A. Nippita ${ }^{1,3}$, Jane B. Ford ${ }^{1}$ and Christine L. Roberts ${ }^{1}$
}

\begin{abstract}
Background: Internationally, repeat caesarean sections make the largest contribution to overall caesarean section rates and inter-hospital variation has been reported. The aim of this study was to determine if casemix and hospital factors explain variation in hospital rates of repeat caesarean sections and whether these rates are associated with maternal and neonatal morbidity.
\end{abstract}

Methods: This population-based record linkage study utilised data from New South Wales, Australia between 2007 and 2011. The study population included maternities with any previous caesarean section(s) and were singleton, cephalic and $\geq 37$ weeks' gestation (Robson Group 5). Multilevel regression models were used to examine variation in hospital rates of 'planned repeat caesarean section' and, among women who planned a vaginal birth, 'intrapartum caesarean section'. We assessed associations between risk-adjusted hospital rates of planned and intrapartum caesarean sections and rates of casemix adjusted maternal and neonatal morbidity, postpartum haemorrhage and Apgar score $<7$ at five minutes.

Results: Of 61894 maternities with a previous caesarean section in 81 hospitals, $82.1 \%$ resulted in a caesarean section (72.7\% planned and $9.4 \%$ unplanned intrapartum caesareans) and $17.9 \%$ in vaginal birth. Observed hospital rates of planned caesarean sections ranged from $50.7 \%$ to $98.4 \%$. Overall $49.0 \%$ of between-hospital variation in planned repeat caesarean section rates was explained by patient (17.3 \%) and hospital factors (31.7\%). Increased odds of planned caesarean section were associated with private hospital status and lower hospital propensity for vaginal birth after caesarean. There were no associations between hospital rates of planned repeat caesarean section and adjusted morbidity rates. Among women who intended a vaginal birth, the observed rates of intrapartum caesarean section ranged from $12.9 \%$ to $71.9 \%$. In total, $27.5 \%$ of between-hospital variation in rates of intrapartum caesarean section was explained by patient (19.5\%) and hospital factors (8.0\%). The adjusted morbidity rates differed among hospital intrapartum caesarean section rates, but were influenced by a few hospitals with outlying morbidity rates.

Conclusions: Among women with at least one previous caesarean section, less than half of the variation in hospital caesarean section rates was explained by differences in hospital's patient characteristics and practices. Strategies aimed at modifying caesarean section rates for these women should not affect morbidity rates.

\footnotetext{
* Correspondence: Kathrin.Schemann@sydney.edu.au

${ }^{1}$ Clinical and Population Perinatal Health Research, Kolling Institute of

Medical Research, University of Sydney at Royal North Shore Hospital, St

Leonards, NSW 2065, Australia

${ }^{2}$ NSW Biostatistics Training Program, NSW Ministry of Health, North Sydney,

NSW 2060, Australia

Full list of author information is available at the end of the article
}

\section{Biomed Central}

(c) 2015 Schemann et al. Open Access This article is distributed under the terms of the Creative Commons Attribution 4.0 International License (http://creativecommons.org/licenses/by/4.0/), which permits unrestricted use, distribution, and reproduction in any medium, provided you give appropriate credit to the original author(s) and the source, provide a link to the Creative Commons license, and indicate if changes were made. The Creative Commons Public Domain Dedication waiver (http://creativecommons.org/publicdomain/zero/1.0/) applies to the data made available in this article, unless otherwise stated. 


\section{Background}

Internationally, caesarean section rates have increased by $50 \%$ or more over the last decade, with rates in the USA, UK and Australia peaking at $26.2 \%, 31.3 \%$ and $32.3 \%$, respectively [1-3]. The lack of availability of caesarean section in developing countries contributes to significant preventable maternal and perinatal morbidity and mortality [4]; yet in developed countries, rising caesarean section rates have not been accompanied by improved perinatal outcomes [5]. In 1985, the World Health Organization recommended a rate between $10 \%$ and $15 \%$ [6], and in 2009 acknowledged that it is important for the appropriate parturient to receive the optimal labour interventions, balancing the risks and benefits of each intervention [7].

Caesarean sections for women with at least one previous caesarean section make an important contribution to the historic rise in the overall caesarean section rate $[8,9]$. In high income countries, Group 5 (multiparous women with at least one caesarean section and a single cephalic pregnancy at $\geq 37$ weeks) [10] of the Robson classification for caesarean sections make the largest contribution to overall caesarean section rates [10-13]. This heterogenous group includes women with differing onsets of labour [10,14-17], women with and without a previous vaginal delivery and women with one or more previous caesarean sections $[14,15,18,19]$. To our knowledge, only one previous study examined adjusted hospital caesarean section rates for Robson Group 5 and identified large, unexplained variation between hospital rates for this group, despite adjustment for a limited number of case-mix factors (maternal age, country of birth of the mother, parity, maternal smoking, diabetes and hypertension) [11]. Variation in hospital caesarean section rates can be due to various factors including differences in patient characteristics or preferences, access to care, clinician behavior and hospital culture or policy. With sufficient data available on individual and hospital characteristics, variation due to known casemix and hospital factors can be accounted for, and the remaining 'unexplained' variation quantified. This 'unexplained' variation represents the contribution of unmeasured factors which result in women with similar characteristics having different outcomes depending on hospital of care $[20,21]$. Unexplained variation in practice is important where it influences health care costs without improving outcomes, and raises questions about the appropriateness of particular hospital practices [22]. However, the previous study lacked information about differences in maternal and infant outcomes or evaluation of hospital characteristics that may contribute to the variation. Clinical factors such as offering trial of labour may also contribute to the variation in elective repeat caesarean section rates and subanalysis of this group by onset of labour has been suggested [17]. Therefore, the aims of this study were, among women with at least one previous caesarean section (Robson Group 5), a) to explore variation in hospital rates of planned and intrapartum repeat caesarean section by intended mode of birth; b) to determine whether casemix and hospital factors explain the variation between hospital rates and c) to examine the association between hospital rates of planned and intrapartum caesarean sections with maternal and neonatal morbidity outcomes.

\section{Methods}

\section{Study population}

The study population included multiparous women with at least one previous caesarean section and a single, cephalic pregnancy at $\geq 37$ weeks' gestation (Robson Group 5, [10]), who gave birth in New South Wales (NSW) in 2007 - 2011. NSW is Australia's most populated state with 7 million residents and 95000 births per annum (32\% of all Australian births) [23]. This study was restricted to births occurring in hospitals having at least 50 births per annum and performing at least 10 caesarean sections per annum. The analyses of intrapartum caesarean section rates following a trial of labour were additionally restricted to hospitals with at least 20 women undergoing a trial of labour during the study period to ensure that only hospitals that offered vaginal birth after caesarean section were included, and to provide sufficient data for modeling of the hospital rate of intrapartum caesarean section.

\section{Data sources and sampling}

Data were obtained from two NSW population databases, the Perinatal Data Collection (PDC) and the Admitted Patient Data Collection (APDC). The PDC is a legislated population-based surveillance system covering all live births and stillbirths of at least 20 weeks gestation or 400 grams birth weight in NSW (subsequently referred to as 'births'). Information in the PDC is collected by the attending doctor or midwife and includes maternal characteristics, medical and obstetric information as well as infant outcomes. The APDC represents a census of all NSW public and private hospital discharges and includes patient characteristics and hospitalisation-related information. Up to 20 diagnoses and procedures in each hospital record are coded according to the $10^{\text {th }}$ revision of the International Classification of Disease, Australian Modification (ICD-10-AM) and the Australian Classification of Health Interventions [24]. Trained clinical coders obtain diagnosis and procedure information by reviewing the entire medical record. The NSW Centre for Health Record Linkage carried out probabilistic record linkage between the two databases, 
with linkage proportions over $98 \%$ [25], prior to providing the researchers with de-identified records for analysis.

\section{Outcome variables}

The primary outcomes were hospital rates of 'planned caesarean sections' and, among women who planned a vaginal birth 'intrapartum caesarean sections'. 'Planned caesarean sections' included women who had at least one previous caesarean section and subsequently had a caesarean section, with no labour or went into spontaneous labour before their planned date [26]. Women who planned a vaginal birth included all other women with spontaneous, augmented or induced labour, regardless of eventual mode of delivery.

\section{Explanatory variables}

The available explanatory variables were categorised into case-mix factors (Table 1) and hospital factors. Maternal age was treated as a continuous variable in analyses. Two area-based variables, socio-economic status using the index of education and occupation based on 2011 census data [27] by postcode of residence and the rate of overweight/obesity based on local health district and year [28] were assigned to each individual record and used as explanatory variables. Hospital factors included birth volume, hospital status (private; public with primary obstetric training; public with secondary obstetric training (large district and rural hospitals that host obstetric registrars); and other (non-training public hospitals)), level of perinatal care (NICU, CPAP, other) and hospital location (urban/rural) [29]. Hospital rates of obstetric transfusions; instrumental birth (forceps and vacuum assisted); caesarean sections performed under general anaesthetic and births where regional analgesia was used (as indicators of anaesthetic services) were also considered. The hospital rate of all hospital births that were low risk births (rate of deliveries of singleton, cephalic pregnancies at term without hypertension, placental conditions, diabetes or other chronic disease and no previous perinatal death, referred to as low risk rate) and hospital usage of oxytocin for induction and/or augmentation among women with previous caesarean section were also used. Additionally, the hospital rate of vaginal delivery for the birth following a primary caesarean section for breech presentation was used as a proxy for hospital predisposition to carry out a vaginal birth after caesarean (referred to as 'hospital propensity towards VBAC'). Variables from the birth record are considered to be reliably reported [30-33] and the coding of hospital diagnoses and procedures has previously been validated [34-37].

\section{Statistical analyses}

Descriptive statistics for continuous variables and frequency tables for categorical variables were used to assess the distribution of the explanatory variables. Mean and standard deviation are reported for normally distributed continuous variables whereas median and inter-quartile ranges are reported for non-normally distributed continuous variables. Observed hospital rates were reported for a) 'planned caesarean sections' (among all women with at least one previous caesarean, Robson Group 5) and b) 'intrapartum caesarean sections' (among all women with at least one previous caesarean who had a trial of labour for a planned vaginal birth).

For each of the two outcomes above, observed hospital rates were compared. Then, multivariable, multilevel binomial logistic regression models were constructed with a manual backward stepwise approach, with a random intercept for hospital to account for clustering of observations from the same hospital and with a shrinkage factor to allow for inclusion of hospitals with small sample size. Models were fitted, progressively adjusting for case-mix and hospital factors, as described previously [38]. Briefly, the first model included only the random hospital effect (hospital intercepts), and produced 'unadjusted rates' accounting only for clustering within hospitals, with rates for smaller hospitals 'shrunken' towards the average rate. Thereafter models were sequentially adjusted for case-mix factors (model 2) and case-mix and hospital factors (model 3).To illustrate the differences in hospital repeat caesarean section rates after each step of adjustment, the risk-adjusted hospital rates with $95 \%$ confidence intervals were plotted. The relative contribution of each step of adjustment to the overall reduction in variation in hospital caesarean section rates was quantified by calculating the difference between the hospital variation of the current and preceding models as a proportion of the unadjusted model's hospital variation.

\section{Assessment of associations of planned and intrapartum hospital caesarean section rates with maternal or neonatal morbidity}

The associations between hospital planned and intrapartum caesarean rates and hospital morbidity rates were assessed. Morbidities included severe maternal and neonatal morbidity, postpartum haemorrhage and infant Apgar score below 7 at five minutes. Severe maternal and neonatal morbidity were determined using validated composite outcome indicators that include both lifethreatening conditions (e.g. respiratory failure, cerebrovascular haemorrhage, shock and cardiac arrest) and procedures associated with severe morbidity (e.g. mechanical ventilation, blood transfusion, acute dialysis and surgical procedures) [35, 39]. Postpartum haemorrhage and Apgar score are accurately reported in these data $[30,40]$. A multilevel logistic regression approach was 
Table 1 Case-mix characteristics of the study population, NSW, 2007-2011. The study population consists of multiparous women with a singleton cephalic-presenting infant at $\geq 37$ weeks gestation with at least one previous caesarean section

\begin{tabular}{|c|c|c|c|c|c|c|c|}
\hline \multicolumn{2}{|l|}{ Variables and levels } & \multirow{2}{*}{$\begin{array}{l}\text { All deliveries } \\
\text { N=61894 (column \%) }\end{array}$} & \multirow{2}{*}{$\begin{array}{l}\text { Any repeat caesarean }^{\text {a }} \\
N=50819 \text { (column \%) }\end{array}$} & \multirow{2}{*}{$\begin{array}{l}\text { Planned repeat CS } \\
\mathrm{N}=45006 \text { (column \%) }\end{array}$} & $\begin{array}{l}\text { Intrapartum repeat CS } \\
\mathrm{N}=5813 \text { (column \%) }\end{array}$ & \multirow{2}{*}{$\begin{array}{l}\text { Vaginal birth after caesarean } \\
N=11075 \text { (column \%) }\end{array}$} & $\begin{array}{l}\text { Planned vaginal birth }^{b} \\
\mathrm{~N}=16888 \text { (column \%) }\end{array}$ \\
\hline \multirow{4}{*}{ Maternal age } & & & & & & & \\
\hline & Under 20 & $314(0.5)$ & $248(0.5)$ & $211(0.5)$ & $37(0.6)$ & $66(0.6)$ & $103(0.6)$ \\
\hline & 20 to 34 & $36626(59.2)$ & $29472(58.0)$ & $25843(57.4)$ & $3629(62.4)$ & $7154(64.6)$ & $10783(63.9)$ \\
\hline & $35+$ & $24954(40.3)$ & $21099(41.5)$ & $18952(42.1)$ & $2147(36.9)$ & $3855(34.8)$ & $6002(35.5)$ \\
\hline \multirow[t]{3}{*}{ Country of birth } & Australia or New Zealand & $45012(72.9)$ & $37018(73.0)$ & $32947(73.4)$ & $4071(70.2)$ & $7994(72.3)$ & $12065(71.6)$ \\
\hline & Europe or North America & $3554(5.8)$ & $2921(5.8)$ & $2575(5.7)$ & $346(6.0)$ & $633(5.7)$ & $979(5.8)$ \\
\hline & Other & $13177(21.3)$ & $10750(21.2)$ & $9368(20.9)$ & $1382(23.8)$ & $2427(22.0)$ & $3809(22.6)$ \\
\hline \multirow[t]{3}{*}{ Model of care } & $\begin{array}{l}\text { Public patient in public } \\
\text { hospital }\end{array}$ & $36059(58.2)$ & $28020(55.1)$ & $24079(53.5)$ & 3941 (67.8) & $8039(72.6)$ & $11980(70.9)$ \\
\hline & $\begin{array}{l}\text { Private patient in public } \\
\text { hospital }\end{array}$ & $5544(9.0)$ & $4667(9.2)$ & $4201(9.3)$ & $466(8.0)$ & $877(7.9)$ & $1343(8.0)$ \\
\hline & $\begin{array}{l}\text { Private patient in private } \\
\text { hospital }\end{array}$ & $20291(32.8)$ & $18132(35.7)$ & $16726(37.2)$ & $1406(24.2)$ & $2159(19.5)$ & $3565(21.1)$ \\
\hline \multicolumn{2}{|l|}{ Smoking in pregnancy } & $6174(10.0)$ & $4599(9.1)$ & $3947(8.8)$ & $652(11.2)$ & $1575(14.2)$ & $2227(13.2)$ \\
\hline \multicolumn{2}{|c|}{ Diabetes (pre-existing or gestational) } & $5233(8.5)$ & $4598(9.0)$ & $4166(9.3)$ & $432(7.4)$ & $635(5.7)$ & $1067(6.3)$ \\
\hline \multicolumn{2}{|l|}{ Hypertensive disorders } & $3327(5.4)$ & $2842(5.6)$ & $2510(5.6)$ & $332(5.7)$ & $485(4.4)$ & $817(4.8)$ \\
\hline \multicolumn{2}{|c|}{ Other chronic medical conditions ${ }^{c}$} & $788(1.3)$ & $709(1.4)$ & $625(1.4)$ & $84(1.4)$ & $79(0.7)$ & $163(1.0)$ \\
\hline \multicolumn{2}{|c|}{ Parity 2 or more (compared to parity 1 ) } & $23911(38.7)$ & $18516(36.5)$ & $17132(38.2)$ & $1384(23.8)$ & $5395(48.8)$ & $6779(40.2)$ \\
\hline \multicolumn{2}{|c|}{2 or more previous caesarean sections } & $13554(22.0)$ & $13353(26.4)$ & $12831(28.6)$ & $522(9.0)$ & $201(1.9)$ & $723(4.3)$ \\
\hline \multicolumn{2}{|c|}{ Labour/vaginal birth prior to first caesarean } & $5819(9.4)$ & $3851(7.6)$ & $3383(7.5)$ & $468(8.1)$ & $1968(17.8)$ & $2436(14.4)$ \\
\hline \multicolumn{2}{|c|}{ Previous vaginal birth after caesarean } & $4130(6.7)$ & $1121(2.2)$ & $834(1.9)$ & $287(4.9)$ & $3009(27.2)$ & $3296(19.5)$ \\
\hline \multicolumn{2}{|c|}{ Previous $3^{\text {rd }}$ or $4^{\text {th }}$ degree perineal tear } & $551(0.9)$ & $402(0.8)$ & $372(0.8)$ & $30(0.5)$ & $149(1.3)$ & $179(1.1)$ \\
\hline \multicolumn{2}{|l|}{ Previous perinatal death } & $1000(1.6)$ & $825(1.6)$ & $752(1.7)$ & $73(1.3)$ & $175(1.6)$ & $248(1.5)$ \\
\hline \multicolumn{2}{|l|}{ Non-CS uterine scar } & $421(0.7)$ & $403(0.8)$ & $377(0.8)$ & $26(0.4)$ & $18(0.2)$ & $44(0.3)$ \\
\hline \multicolumn{2}{|c|}{$\begin{array}{l}\text { Assisted reproductive technology use, last } 12 \\
\text { months }\end{array}$} & 1914 (3.1) & $1594(3.1)$ & $1447(3.2)$ & $147(2.5)$ & $320(2.9)$ & $467(2.8)$ \\
\hline \multirow{4}{*}{$\begin{array}{l}\text { Inter-pregnancy interval } \\
\text { (months) }\end{array}$} & $0-17$ & $21126(34.1)$ & $17082(33.6)$ & $15136(33.6)$ & $1946(33.5)$ & $4044(36.5)$ & $5990(35.5)$ \\
\hline & $18-59$ & $27761(44.9)$ & $23069(45.4)$ & $20617(45.8)$ & $2452(42.2)$ & $4692(42.4)$ & $7144(42.3)$ \\
\hline & $60+$ & $4903(7.9)$ & $4066(8.0)$ & $3605(8.0)$ & $461(7.9)$ & $837(7.6)$ & $1298(7.7)$ \\
\hline & Unknown & $8104(13.1)$ & $6602(13.0)$ & $5648(12.5)$ & $954(16.4)$ & $1502(13.6)$ & $2456(14.5)$ \\
\hline \multicolumn{2}{|l|}{ Placental conditions ${ }^{d}$} & $1079(1.7)$ & $990(1.9)$ & $807(1.8)$ & $183(3.1)$ & $89(0.8)$ & $272(1.6)$ \\
\hline \multirow[t]{2}{*}{ Gestational age (weeks) } & 37 & $4597(7.4)$ & $3870(7.6)$ & $3320(7.4)$ & $550(9.5)$ & $727(6.6)$ & $1277(7.6)$ \\
\hline & 38 & 19452 (31.4) & 17579 (34.6) & 16389 (36.4) & $1190(20.5)$ & $1873(16.9)$ & 3063 (18.1) \\
\hline
\end{tabular}


Table 1 Case-mix characteristics of the study population, NSW, 2007-2011. The study population consists of multiparous women with a singleton cephalic-presenting infant at $\geq 37$ weeks gestation with at least one previous caesarean section (Continued)

\begin{tabular}{|c|c|c|c|c|c|c|c|}
\hline & 39 & $25143(40.6)$ & $21915(43.1)$ & $20366(45.3)$ & $1549(26.6)$ & $3228(29.1)$ & $4777(28.3)$ \\
\hline & 40 & $9016(14.6)$ & $5367(10.6)$ & $3782(8.4)$ & $1585(27.3)$ & $3649(32.9)$ & $5234(31.0)$ \\
\hline & $41+$ & $3686(6.0)$ & $2088(4.1)$ & $1149(2.6)$ & $939(16.2)$ & $1598(14.4)$ & $2537(15.0)$ \\
\hline \multirow{3}{*}{$\begin{array}{l}\text { Birth weight for gestational } \\
\text { age }\end{array}$} & Small $\left(<10^{\text {th }}\right.$ percentile $)$ & $5,501(8.9)$ & $4110(8.1)$ & $3501(7.8)$ & $609(10.5)$ & $1391(12.6)$ & $2000(11.8)$ \\
\hline & Appropriate & $49453(79.9)$ & $40540(79.8)$ & $35962(79.9)$ & $4578(78.8)$ & $8913(80.5)$ & $13491(79.9)$ \\
\hline & Large $\left(>90^{\text {th }}\right.$ percentile) & $6927(11.2)$ & $6158(12.1)$ & $5533(12.3)$ & $625(10.8)$ & $769(6.9)$ & $1394(8.3)$ \\
\hline \multicolumn{2}{|l|}{ Stilllbirth } & $60(0.1)$ & $30(0.1)$ & $23(0.1)$ & $7(0.1)$ & $30(0.3)$ & $37(0.2)$ \\
\hline \multicolumn{2}{|l|}{ Severe maternal morbidity } & $654(1.9)$ & $526(1.9)$ & $395(1.6)$ & $131(4.0)$ & $128(2.1)$ & $259(2.8)$ \\
\hline \multicolumn{2}{|l|}{ Severe neonatal morbidity } & $1275(2.1)$ & $1058(2.1)$ & $851(1.9)$ & $207(3.6)$ & $217(2.0)$ & $424(2.5)$ \\
\hline \multicolumn{2}{|l|}{ Postpartum haemorrhage } & $2847(4.6)$ & $1666(3.3)$ & $1301(2.9)$ & $365(6.3)$ & $1181(10.7)$ & $1546(9.2)$ \\
\hline \multicolumn{2}{|c|}{ Apgar score at five minutes $<7$} & $520(0.8)$ & $371(0.7)$ & $261(0.6)$ & $110(1.9)$ & $149(1.4)$ & $259(1.5)$ \\
\hline
\end{tabular}

$\mathrm{CS}=$ caesarean section

(n)

${ }^{b}$ Includes intrapartum repeat caesarean section and vaginal birth after caesarean section

Included renal, cardiac, asthma/COPD, autoimmune, thyroid and inflammatory bowel disease

${ }^{d}$ Included morbidly adherent placenta, placental abruption, placenta praevia, antepartum haemorrhage 
used. Hospitals were divided into quintiles according to their risk-adjusted caesarean section rates. The quintiles were then used as an additional categorical variable to predict case-mix adjusted morbidity rates. Case-mix adjusted hospital rates of maternal and neonatal morbidity within each caesarean section rate quintile were then averaged and the estimated adjusted odds ratios of morbidity for each quintile were compared. The riskadjusted hospital caesarean section rates were also plotted against the case-mix adjusted rates of maternal and neonatal morbidity.

All statistical analyses were conducted using SAS Enterprise Guide statistical software (release 5.1 ( ) 2012, SAS Institute Inc., Cary, NC, USA).

This study was approved by the NSW Population and Health Services Research Ethics Committee.

\section{Results}

From 2007 to 2011 there were 63316 singleton cephalic, term births in NSW among mothers with at least one previous caesarean section (Robson Group 5). Among all maternities, the proportions of births in Robson Group 5 increased from $12.7 \%$ in 2007 to $14.1 \%$ in 2011. After exclusions (739 from ineligible hospitals and 683 with missing data), 61894 maternities from 81 hospitals were included in the analysis. Overall, there were 51388 (82.1\%) women who had a further caesarean section; including 45006 (72.7 \%) deliveries with a planned caesarean section. Of the 16888 women intending to have a vaginal birth, $34.4 \%$ had an intrapartum caesarean section and $65.6 \%$ had a vaginal birth.

The distributions of key characteristics among women with at least one previous caesarean section are presented in Table 1. Women with a planned caesarean section tended to be older, private patients, have more medical and obstetric conditions and two or more previous caesarean sections than women with a trial of labour. Among women with a trial of labour for a planned vaginal birth, those who had intrapartum caesarean sections were more likely to be private patients, (a) Unadjusted random intercept model

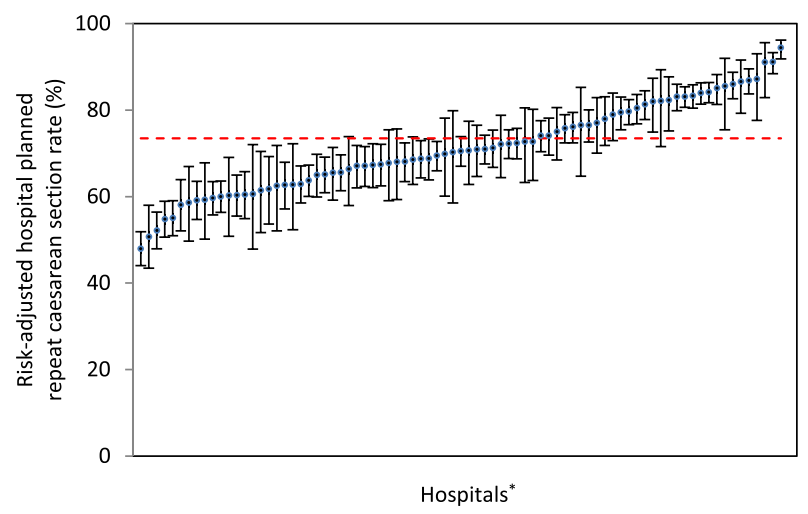

(c) Adjusted for case-mix and hospital factors

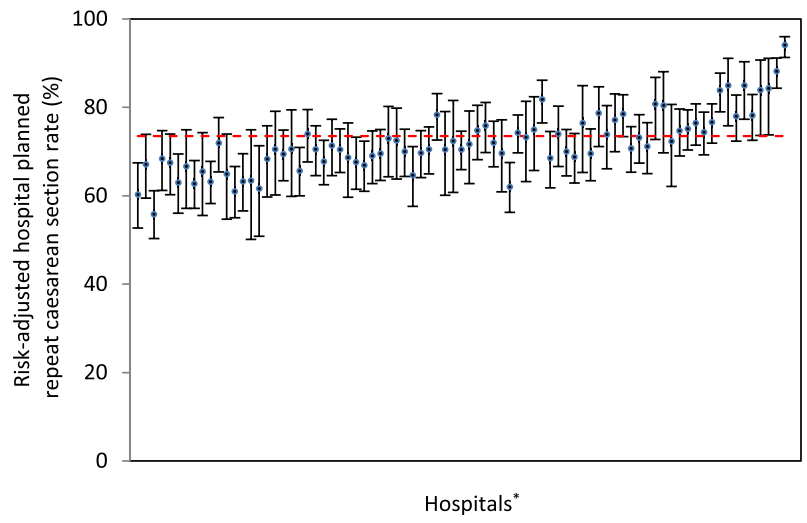

(b) Adjusted for case-mix

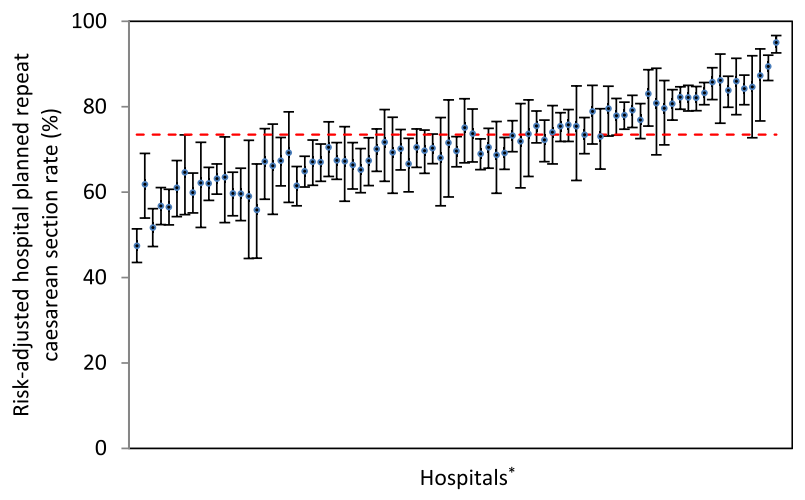

Fig. 1 Hospital planned repeat caesarean section rates in NSW, 2007-2011. * The data points represent the rates of planned repeat caesarean section among multiparous women with a singleton, cephalic-presenting infant of $\geq 37$ weeks gestation in 81 hospitals, ordered from lowest to highest unadjusted hospital CS rate. The vertical error bars indicate the $95 \%$ confidence intervals for each estimate. Dashed horizontal lines indicate the mean risk-adjusted planned caesarean section rate 
Table 2 Predictive factors for planned repeat caesarean section, NSW, 2007-2011. Two models were fitted progressively adjusting for case-mix and hospital factors

\begin{tabular}{|c|c|c|}
\hline \multirow[t]{2}{*}{ Variable and categories } & Case-mix model & Hospital model \\
\hline & OR $(95 \% \mathrm{Cl})$ & OR $(95 \% \mathrm{Cl})$ \\
\hline Maternal age (years) & $1.02(1.02,1.03)$ & $1.02(1.02,1.03)$ \\
\hline \multicolumn{3}{|l|}{ Country of birth } \\
\hline Australia or New Zealand & 1.00 & 1.00 \\
\hline Europe or North America & $0.83(0.76,0.91)$ & $0.83(0.76,0.91)$ \\
\hline Other & $0.87(0.82,0.93)$ & $0.88(0.83,0.93)$ \\
\hline Smoking & $0.91(0.85,0.98)$ & $0.92(0.85,0.99)$ \\
\hline \multicolumn{3}{|l|}{ Socio-economic status ${ }^{a}$} \\
\hline 1st quintile (high) & $1.27(1.14,1.42)$ & $1.28(1.15,1.43)$ \\
\hline 2nd quintile & $1.17(1.06,1.30)$ & $1.18(1.07,1.31)$ \\
\hline 3rd quintile & $1.18(1.07,1.30)$ & $1.18(1.07,1.30)$ \\
\hline 4th quintile & $1.01(0.93,1.10)$ & $1.01(0.93,1.10)$ \\
\hline 5th quintile (low) & 1.00 & 1.00 \\
\hline $\begin{array}{l}\text { Diabetes (pre-existing or } \\
\text { gestational) }\end{array}$ & $1.27(1.17,1.38)$ & $1.28(1.18,1.39)$ \\
\hline Hypertensive disorders & $1.10(1.00,1.21)$ & $1.11(1.01,1.22)$ \\
\hline $\begin{array}{l}\text { Other chronic medical } \\
\text { conditions }^{\mathrm{b}}\end{array}$ & $1.42(1.16,1.74)$ & $1.43(1.17,1.74)$ \\
\hline Placental conditions $^{c}$ & $1.11(0.94,1.30)$ & $1.11(0.94,1.30)$ \\
\hline $\begin{array}{l}\text { Parity } 2 \text { or more } \\
\text { (versus parity } 1 \text { ) }\end{array}$ & $2.09(1.97,2.22)$ & $2.10(1.98,2.22)$ \\
\hline $\begin{array}{l}\text { Labour/vaginal birth prior } \\
\text { to index caesarean }\end{array}$ & $0.28(0.26,0.31)$ & $0.28(0.26,0.31)$ \\
\hline $\begin{array}{l}\text { Previous vaginal birth after } \\
\text { caesarean }\end{array}$ & $0.05(0.04,0.05)$ & $0.05(0.04,0.05)$ \\
\hline $\begin{array}{l}\text { Previous } 3^{\text {rd }} \text { or } 4^{\text {th }} \text { degree } \\
\text { perineal tear }\end{array}$ & $2.44(1.93,3.07)$ & $2.44(1.93,3.07)$ \\
\hline Previous perinatal death & $2.66(2.20,3.21)$ & $2.66(2.20,3.20)$ \\
\hline Non-CS uterine scar & $2.20(1.55,3.12)$ & $2.21(1.56,3.13)$ \\
\hline $\begin{array}{l}\text { Assisted reproductive } \\
\text { technology use } \\
\text { (last } 12 \text { months) }\end{array}$ & $0.81(0.72,0.91)$ & $0.81(0.71,0.91)$ \\
\hline \multicolumn{3}{|l|}{$\begin{array}{l}\text { Inter-pregnancy interval } \\
\text { (months) }\end{array}$} \\
\hline $0-17$ & $0.90(0.86,0.95)$ & $0.90(0.86,0.95)$ \\
\hline $18-59$ & 1.00 & 1.00 \\
\hline $60+$ & $1.05(0.96,1.14)$ & $1.05(0.96,1.14)$ \\
\hline Unknown & $0.69(0.64,0.73)$ & $0.69(0.64,0.73)$ \\
\hline \multicolumn{3}{|l|}{$\begin{array}{l}\text { Estimated gestational } \\
\text { age (weeks) }\end{array}$} \\
\hline 37 & $0.57(0.52,0.62)$ & $0.57(0.52,0.62)$ \\
\hline 38 & $1.14(1.08,1.21)$ & $1.14(1.08,1.21)$ \\
\hline 39 & 1.00 & 1.00 \\
\hline 40 & $0.18(0.17,0.20)$ & $0.18(0.17,0.20)$ \\
\hline \multirow[t]{2}{*}{$41+$} & $0.14(0.12,0.15)$ & $0.14(0.12,0.15)$ \\
\hline & $0.77(0.71,0.84)$ & $0.77(0.71,0.84)$ \\
\hline
\end{tabular}

Table 2 Predictive factors for planned repeat caesarean section, NSW, 2007-2011. Two models were fitted progressively adjusting for case-mix and hospital factors (Continued)

Small for gestational age

$\left(<10^{\text {th }}\right.$ percentile)

Large for gestational age $\left(>90^{\text {th }}\right.$ percentile)

Stillbirth

$\begin{array}{ll}1.47(1.38,1.57) & 1.47(1.38,1.57) \\ 0.24(0.13,0.46) & 0.25(0.13,0.46) \\ - & 0.95(0.93,0.96)\end{array}$

Repeat caesarean rate after nulliparous breech caesarean (Propensity towards vaginal birth after caesarean section)

Oxytocin induction and/or augmentation for women with previous caesarean

Low risk rate among all birth $0.95(0.90,1.01)$ Level of hospital care

Public with primary training $\quad 1.00$

Public with secondary training $\quad-\quad 1.30(0.91,1.85)$

$\begin{array}{ll}\text { Public, other } \quad \text { - } & 1.28(0.89,1.82)\end{array}$

Private

$1.80(1.24,2.61)$

Index of Education and Occupation published by the Australian Bureau of Statistics based on postcode [27]

' Included renal, cardiac, asthma/c obstructive pulmonary, autoimmune,

thyroid and inflammatory bowel disease

Included morbidly adherent placenta, placental abruption, placenta praevia,

antepartum haemorrhage

have two or more previous caesarean sections and have not previously experienced labour or vaginal birth before their first caesarean or a successful vaginal birth after caesarean compared to women with vaginal birth (Table 1).

Of the 81 hospitals, $16(19.8 \%)$ were private as opposed to public, 47 (58.0\%) were regional as opposed to metropolitan and $31(38.3 \%)$ provided primary or secondary obstetric training as opposed to not providing obstetric training. Over the study period, the median annual hospital volume of singleton cephalic deliveries at term by multiparous women with a previous caesarean scar was 119 (Inter quartile range: 31-223). The mean hospital instrumental birth rate for all maternities with labour was $14.6 \%$ (standard deviation: $5.6 \%$ ). The mean hospital rate of propensity towards VBAC was $63.2 \%$ (standard deviation: $19.2 \%$ ).

\section{Variation in hospital planned caesarean section rates}

Among women with at least one previous caesarean, the observed hospital planned caesarean section rates for the 81 hospitals ranged from $50.7 \%$ to $98.4 \%$. The unadjusted model had hospital rates ranging from $47.9 \%$ to $94.4 \%$ and 52 of 81 hospitals had rates that differed from the state average (unadjusted model; Fig. 1a). After adjusting for case-mix factors, the unexplained variation between 
hospitals was reduced by $17.3 \%$ with the adjusted hospital planned caesarean section rates ranging from $47.5 \%$ to 95.0 \% (Fig. 1b). However, 42 of 81 hospitals differed from the state average. Maternal medical and prior pregnancy complications were positively associated with planned caesarean section (Table 2). Further adjustment using hospital-level factors explained an additional $31.7 \%$ of the variation between hospital planned caesarean section rates, with adjusted rates ranging from $55.8 \%$ to $94.1 \%$ (Fig. 1c; Table 2). Birthing in hospitals that were private, had low propensity towards VBAC and had a high rate of low risk maternities was associated with higher odds of planned caesarean section (Table 2). There was a tendency for hospitals that used oxytocin to induce and/or augment women with a previous caesarean section to have lower odds of planned caesarean section (OR: 0.95, 95 \% CI: 0.90, 1.01; Table 2). Overall the final model adjusting for casemix and hospital factors explained $49.0 \%$ of the variation between hospital planned repeat caesarean section rates, mostly due to hospital factors, but 23 of 81 hospitals had adjusted rates that differed from the state average.

\section{Variation in hospital intrapartum caesarean section rates following a trial of labour}

Among women with at least one previous caesarean who had a trial of labour for a planned vaginal birth, the observed hospital rates of intrapartum caesarean section ranged from $12.9 \%$ to $71.9 \%$. Unadjusted hospital rates of intrapartum caesarean section following a trial of labour ranged from $21.9 \%$ to $55.3 \%$ and 20 of 73 hospitals had rates that differed from the state average (Fig. 2a). After adjusting for case-mix factors, the unexplained variation between hospitals was reduced by $19.5 \%$ with the adjusted hospital intrapartum caesarean section rates ranging from $22.2 \%$ to $50.9 \%$ and 16 of 73 hospitals being different from the state average (Fig. 2b). Additional adjustment for hospital factors further reduced the unexplained variation by $8.0 \%$ with adjusted rates ranging from $22.5 \%$ to $50.2 \%$, Fig. 2c). The details of these models are presented in Table 3. Hospitals with generally higher instrumental delivery rates had lower odds of having intrapartum caesarean section (OR: 0.82, 95 \% CI: 0.71, 0.93; Table 3). Overall the final model (a) Unadjusted random intercept model

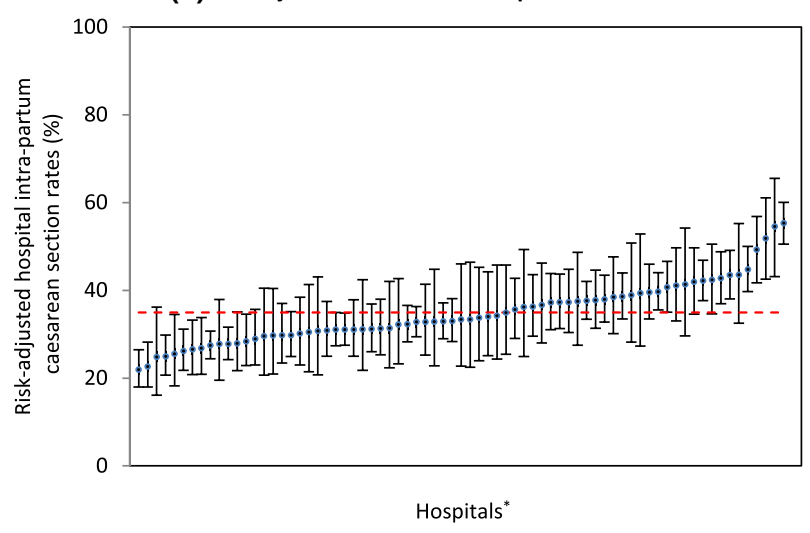

(c) Adjusted for case-mix and hospital factors

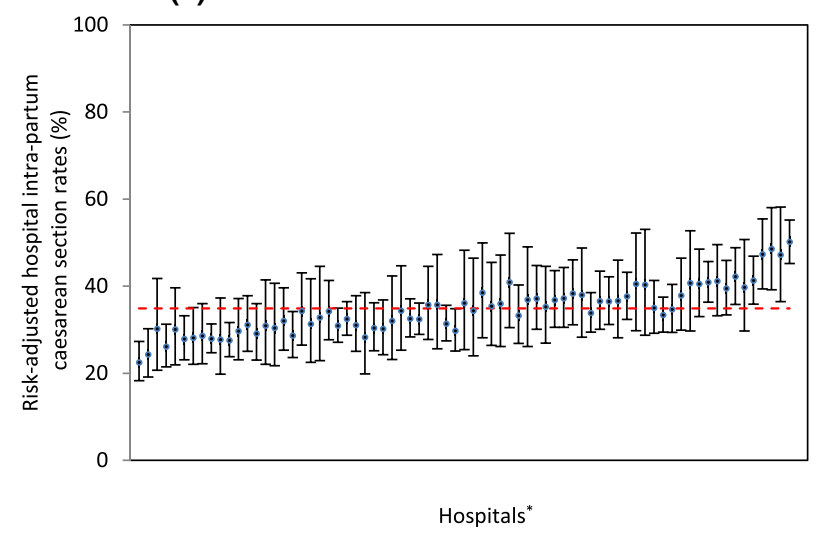

(b) Adjusted for case-mix

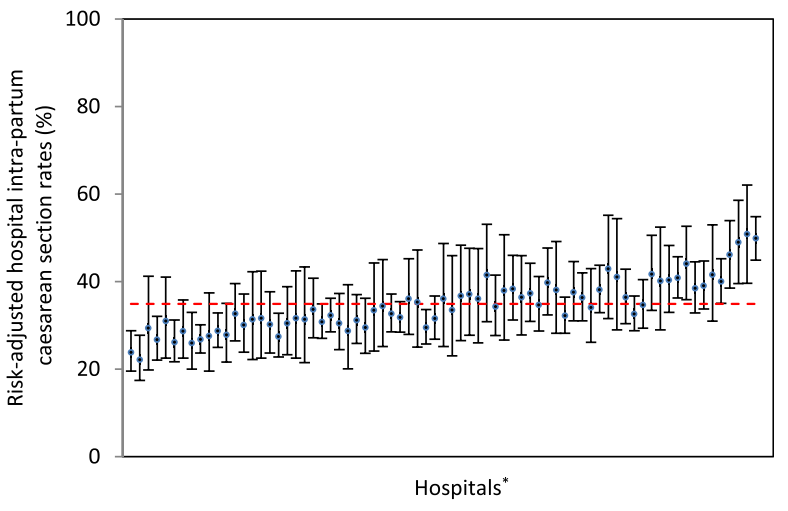

Fig. 2 Hospital intrapartum repeat caesarean section rates in NSW, 2007-2011. * The data points represent the rates of intrapartum repeat caesarean section among multiparous women with a singleton, cephalic-presenting infant of $\geq 37$ weeks gestation undergoing a trial of labour in 73 hospitals, ordered from lowest to highest unadjusted hospital CS rate. The vertical error bars indicate the $95 \%$ confidence intervals for each estimate. Dashed horizontal lines indicate the mean risk-adjusted planned caesarean section rates 
Table 3 Predictive factors for intrapartum caesarean section among women with a trial of labour for a planned vaginal birth, NSW, 2007-2011. Two models were fitted progressively adjusting for case-mix and hospital factors

\begin{tabular}{|c|c|c|}
\hline \multirow[t]{2}{*}{ Variable and categories } & Case-mix model & Hospital model \\
\hline & OR $(95 \% \mathrm{Cl})$ & OR $(95 \% \mathrm{Cl})$ \\
\hline Maternal age (years) & $1.02(1.01,1.03)$ & $1.02(1.02,1.03)$ \\
\hline \multicolumn{3}{|l|}{ Country of birth } \\
\hline Australia or New Zealand & 1.00 & 1.00 \\
\hline Europe or North America & $0.86(0.74,1.00)$ & $0.86(0.74,1.00)$ \\
\hline Other & $1.17(1.06,1.29)$ & $1.17(1.06,1.29)$ \\
\hline Smoking & $1.15(1.03,1.29)$ & $1.14(1.02,1.29)$ \\
\hline Diabetes (pre-existing or gestational) & $1.27(1.10,1.46)$ & $1.27(1.10,1.46)$ \\
\hline Hypertensive disorders & $1.31(1.12,1.53)$ & $1.31(1.12,1.53)$ \\
\hline Other chronic medical conditions ${ }^{\mathrm{a}}$ & $1.95(1.38,2.73)$ & $1.93(1.37,2.71)$ \\
\hline Parity 2 or more (versus parity 1) & $0.70(0.63,0.78)$ & $0.70(0.63,0.78)$ \\
\hline $\begin{array}{l}\text { Labour/vaginal birth prior to index } \\
\text { caesarean }\end{array}$ & $0.48(0.42,0.56)$ & $0.48(0.42,0.55)$ \\
\hline $\begin{array}{l}\text { Previous vaginal birth after } \\
\text { caesarean }\end{array}$ & $0.16(0.14,0.19)$ & $0.16(0.14,0.19)$ \\
\hline Previous perinatal death & $1.80(1.32,2.46)$ & $1.79(1.31,2.44)$ \\
\hline Non-CS uterine scar & $2.78(1.41,5.48)$ & $2.75(1.39,5.44)$ \\
\hline $\begin{array}{l}\text { Assisted reproductive technology } \\
\text { use (last } 12 \text { months) }\end{array}$ & $0.55(0.44,0.68)$ & $0.55(0.45,0.68)$ \\
\hline \multicolumn{3}{|l|}{ Inter-pregnancy interval (months) } \\
\hline $0-17$ & $0.92(0.85,1.00)$ & $0.92(0.85,1.00)$ \\
\hline $18-59$ & 1.00 & 1.00 \\
\hline $60+$ & $1.22(1.06,1.40)$ & $1.21(1.06,1.40)$ \\
\hline Unknown & $0.83(0.74,0.92)$ & $0.82(0.74,0.92)$ \\
\hline Placental conditions ${ }^{b}$ & $4.26(3.22,5.65)$ & $4.26(3.22,5.65)$ \\
\hline \multicolumn{3}{|l|}{ Estimated gestational age (weeks) } \\
\hline 37 & $1.58(1.37,1.82)$ & $1.58(1.38,1.82)$ \\
\hline 38 & $1.30(1.18,1.44)$ & $1.30(1.18,1.45)$ \\
\hline 39 & 1.00 & 1.00 \\
\hline 40 & $0.94(0.86,1.03)$ & $0.94(0.86,1.03)$ \\
\hline $41+$ & $1.41(1.26,1.58)$ & $1.41(1.26,1.57)$ \\
\hline $\begin{array}{l}\text { Large for gestational age } \\
\left(>90^{\text {th }} \text { percentile) }\right.\end{array}$ & $1.71(1.53,1.90)$ & $1.71(1.53,1.91)$ \\
\hline Stillbirth & $0.26(0.11,0.62)$ & $0.25(0.10,0.62)$ \\
\hline $\begin{array}{l}\text { Repeat caesarean rate after } \\
\text { nulliparous breech caesarean } \\
\text { (Propensity towards vaginal } \\
\text { birth after caesarean section) }\end{array}$ & - & $1.04(1.02,1.07)$ \\
\hline Instrumental delivery rate & - & $0.82(0.71,0.93)$ \\
\hline
\end{tabular}

Included renal, cardiac, asthma/COPD, autoimmune, thyroid and inflammatory bowel disease

${ }^{\mathrm{b}}$ Included morbidly adherent placenta, placental abruption, placenta praevia, antepartum haemorrhage explained $27.5 \%$ of the variation between hospital intrapartum caesarean section rates but 14 of 73 hospitals differed from the state average.

\section{Associations with maternal and neonatal morbidities}

Overall, when compared to planned caesarean sections, morbidity rates (severe maternal and neonatal morbidity, postpartum haemorrhage and Apgar score at five minutes less than 7) were higher for women undergoing a trial of labour for a planned vaginal birth (Table 1). Highest rates of adverse outcomes were primarily among women with an intrapartum caesarean section, with the notable exception of postpartum haemorrhage which was highest among women with a vaginal birth. However, in Australia the threshold for postpartum haemorrhage is lower $(500 \mathrm{ml})$ for vaginal birth than for caesarean section $(750 \mathrm{ml})[24]$. For planned repeat caesarean sections, case-mix adjusted hospital rates of severe maternal morbidity, ranged from $1.4 \%$ to $2.8 \%$ (Fig. 3a) and case-mix adjusted hospital rates of severe neonatal morbidity ranged from $1.1 \%$ to 4.7 \% (Fig. 4a). Adjusted hospital rates of postpartum haemorrhage ranged from $0.2 \%$ to $3.3 \%$ for all births (Fig. 5a) and adjusted rates of Apgar score below 7 at five minutes ranged from $0.4 \%$ to $2.2 \%$ (Fig. 6a). There were no associations between hospital planned caesarean section rate quintiles and any of the morbidity measures and no specific patterns indicated in the scatter plots (Figs. 3a, 4a, 5a, 6a; Table 4).

For births following a trial of labour, adjusted hospital rates of severe maternal and neonatal morbidity ranged from $2.3 \%$ to $3.8 \%$ and $1.5 \%$ to $5.2 \%$, respectively (Figs. 3b and $4 \mathrm{~b}$ ). Hospital rates of postpartum haemorrhage, adjusted for case-mix factors, ranged from $6.7 \%$ to $16.0 \%$ for all deliveries following a trial of labour (Fig. 5b). The adjusted maternal morbidity rate was higher for quintile 2 of the hospital intrapartum caesarean section rates when compared to quintile 3 , whereas adjusted neonatal morbidity and postpartum haemorrhage rates were lower for quintiles 1,4 and 5 compared to quintile 3 of hospital intrapartum caesarean section rates (Table 5). A similar trend was observed for adjusted rates of Apgar score below 7 at five minutes (Fig. 6b). A few hospitals with outlying case-mix adjusted morbidity rates caused the differences in morbidity rates among quintiles of hospital intrapartum caesarean section rates (Figs. $4 \mathrm{~b}$ and $5 \mathrm{~b}$ ).

\section{Discussion}

Overall in NSW between 2007 and 2011, $82.1 \%$ of women with at least one previous caesarean section and a singleton, cephalic infant at term had a further caesarean section, and for the majority of women it was a planned caesarean section (72.7 \%). A trial of labour was 

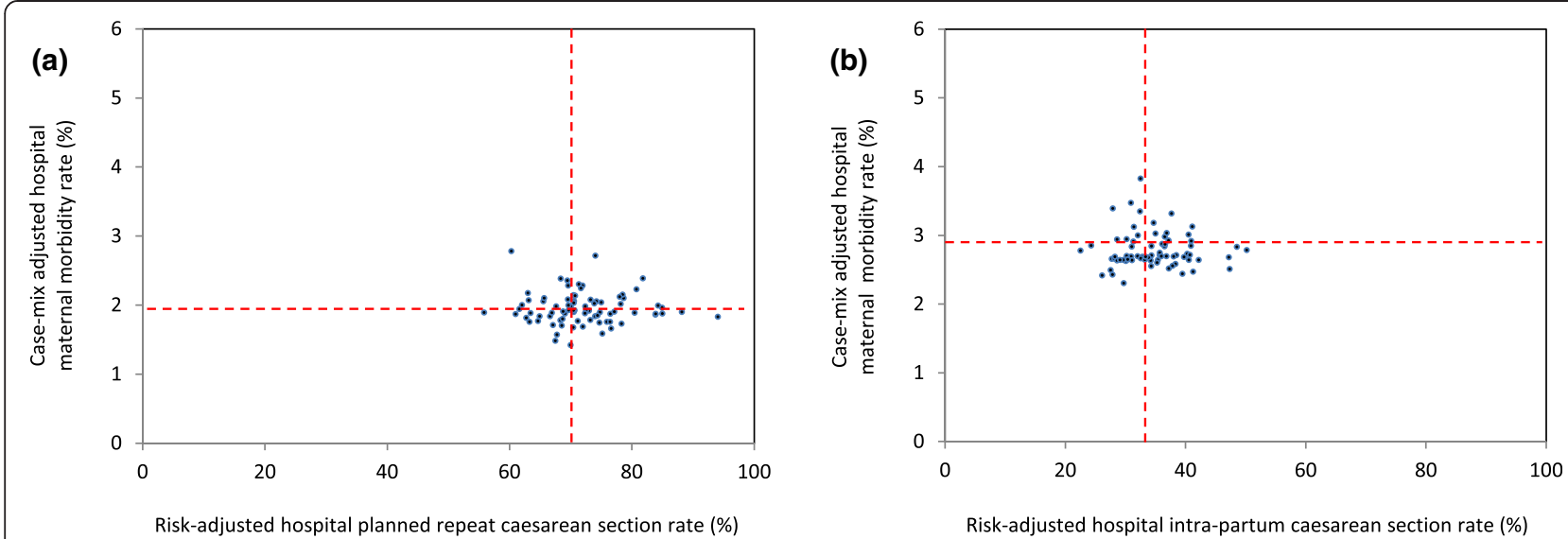

Fig. 3 Severe maternal morbidity and risk-adjusted hospital (a) planned and (b) intrapartum caesarean section rates. Dashed lines indicate the respective mean risk-adjusted caesarean and case-mix adjusted morbidity rates. The study population consisted of multiparous women with a previous caesarean section and a singleton, cephalic-presenting infant of $\geq 37$ weeks gestation in NSW, 2007-2011

relatively uncommon $(27.3 \%)$, although $65.6 \%$ of those women attempting a trial of labour had a successful vaginal birth after caesarean. This finding suggests that even though not many women are selected for a trial of labour, the ones selected are good candidates for vaginal birth after caesarean. Consistent with other studies, both analyses in this study found that the strongest predictive factors for a vaginal birth after caesarean were having a previous vaginal birth after caesarean and having experienced labour/vaginal birth before the index caesarean [41]. Case-mix and hospital factors explained about half (49\%) of the variation in hospital rates of planned repeat caesarean sections and just over a quarter (27.5\%) of between hospital variation in intrapartum caesarean section rates. After final adjustment, 23 of 81 (28.4\%) and 14 of $73(19.2 \%)$ of hospitals had rates that differed from the state average for planned and intrapartum repeat caesarean sections, respectively. There were no associations between quintiles of hospital planned caesarean section rates and morbidity outcomes, but four outlying observations resulted in some associations between quintiles of hospital intrapartum caesarean section rates and morbidity outcomes. A triangular pattern was evident, with the second and third quintiles of hospital intrapartum caesarean section rates having the highest morbidity rates.

To our knowledge this is the first study to examine the variation in caesarean section rates among multiparous women with at least one previous caesarean section with a singleton cephalic fetus at term by intended mode of birth and to explore their maternal and neonatal outcomes. This study utilised large, linked population health datasets with reliably identified and validated variables used for risk adjustment. Multi-level modelling accounted for similarities of births within hospitals and inclusion of a shrinkage factor allowed inclusion of hospitals with small
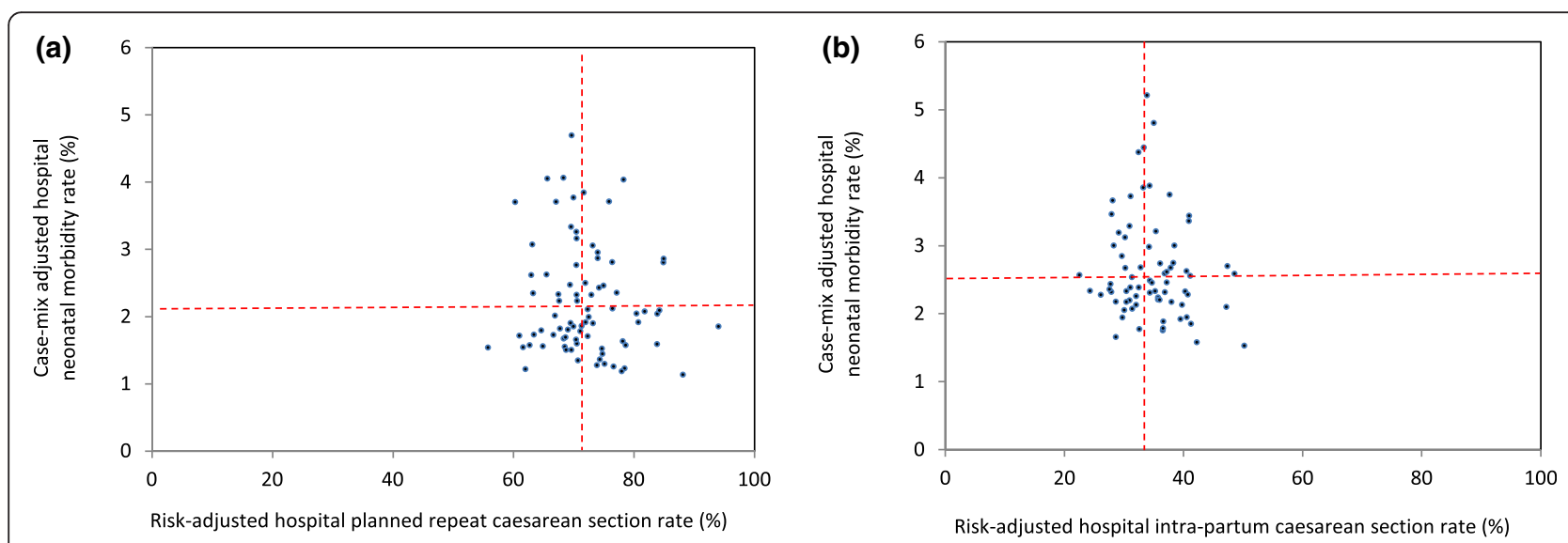

Fig. 4 Severe neonatal morbidity and risk-adjusted hospital (a) planned and (b) intrapartum caesarean section rates. Dashed lines indicate the respective mean risk-adjusted caesarean and case-mix adjusted morbidity rates. The study population consisted of multiparous women with a previous caesarean section and a singleton, cephalic-presenting infant of $\geq 37$ weeks gestation in NSW, 2007-2011 

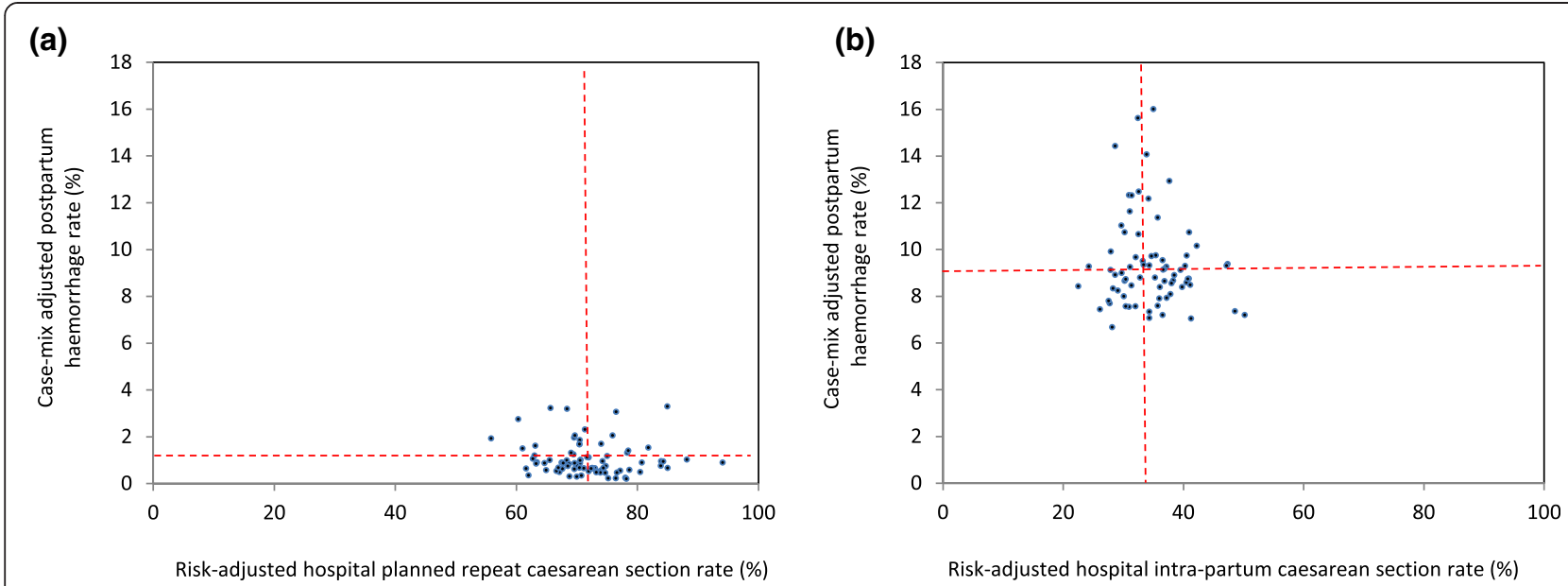

Fig. 5 Postpartum haemorrhage and risk-adjusted hospital (a) planned and (b) intrapartum caesarean section rates. Dashed lines indicate the respective mean risk-adjusted caesarean and case-mix adjusted morbidity rates. The study population consisted of multiparous women with a previous caesarean section and a singleton, cephalic-presenting infant of $\geq 37$ weeks gestation in NSW, 2007-2011

sample size. However, limitations include the lack of information on individual patient attitudes and physician decision-making processes. Different hospital reporting practices may also contribute to the observed hospital variation and this could not be further investigated in this study. Analyses were also restricted to area-based measures of body mass index and socio-economic status as this data is not currently available for individual patients.

The large variation in hospital rates of planned and intrapartum caesarean section found for Robson Group 5 in this study is consistent with previous work finding substantial inter-institutional variation in the mode of birth for deliveries among women with previous caesarean section and/or uterine surgery and a singleton pregnancy [42]. However, direct comparison of birth outcomes with this study is not possible due to differing definitions of a trial of labour and the lack of restriction to cephalic presenting term births.

Casemix heterogeneity within Robson Group 5 [10, 14, $15,17]$ was hypothesised to substantially contribute to the between hospital variation. However, analysis according to the onset of labour and adjusting for case-mix factors including obstetric history, prior experience of labour, and prior vaginal birth and/or vaginal birth after caesarean only somewhat reduced the overall variation in planned repeat caesarean section rates (17.3\%). Although differences in casemix may be important in explaining variation in hospital caesarean rates, these findings suggest that hospital planned repeat caesarean section rates vary markedly for reasons other than individual's characteristics. This finding is consistent with previous studies examining variation in caesarean section
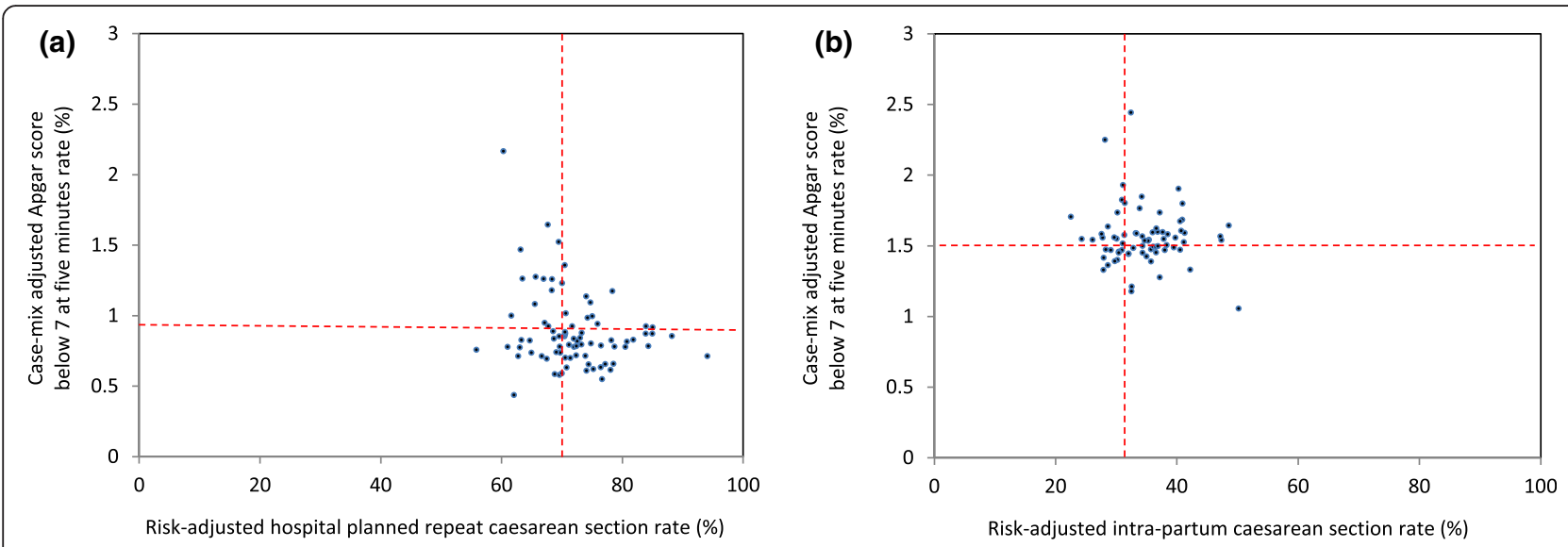

Fig. 6 Apgar score below 7 at five minutes and risk-adjusted hospital (a) planned and (b) intrapartum caesarean section rates. Dashed lines indicate the respective mean risk-adjusted caesarean and case-mix adjusted morbidity rate. The study population consisted of multiparous women with a previous caesarean section and a singleton, cephalic-presenting infant of $\geq 37$ weeks gestation in NSW, 2007-2011 
Table 4 Case-mix adjusted morbidity rates across quintiles of hospital planned caesarean section rates in NSW, 2007-2011

\begin{tabular}{|c|c|c|c|c|c|c|c|c|}
\hline \multirow[t]{2}{*}{ Hospital quintiles } & \multicolumn{2}{|c|}{ Maternal morbidity } & \multicolumn{2}{|c|}{ Neonatal morbidity } & \multicolumn{2}{|c|}{ Postpartum haemorrhage } & \multicolumn{2}{|c|}{ Apgar score at 5 minutes $<7$} \\
\hline & Rate $(95 \% \mathrm{Cl})$ & OR $(95 \% \mathrm{Cl})$ & Rate (95\% Cl) & OR $(95 \% \mathrm{Cl})$ & Rate (95 \% Cl) & OR $(95 \% \mathrm{Cl})$ & Rate $(95 \%$ Cl) & OR $(95 \% \mathrm{Cl})$ \\
\hline \multicolumn{9}{|c|}{ Planned repeat caesarean } \\
\hline 1st quintile (low) & $2.1(1.8,2.4)$ & $0.96(0.70,1.32)$ & $2.4(1.4,3.4)$ & $0.86(0.58,1.29)$ & $1.6(0.7,2.5)$ & $1.16(0.69,1.96)$ & $1.1(0.6,1.7)$ & $0.96(0.70,1.32)$ \\
\hline 2nd quintile & $1.9(1.5,2.2)$ & $0.78(0.57,1.08)$ & $2.2(1.4,3.1)$ & $0.97(0.66,1.43)$ & $1.2(0.2,2.1)$ & $0.97(0.58,1.62)$ & $0.9(0.6,1.2)$ & $0.78(0.57,1.08)$ \\
\hline 3rd quintile & $2.1(1.8,2.3)$ & 1.00 & $2.4(1.7,3.1)$ & 1.00 & $1.1(0.6,1.6)$ & 1.00 & $0.9(0.7,1.1)$ & 1.00 \\
\hline 4th quintile & $1.9(1.5,2.3)$ & $0.84(0.61,1.14)$ & $2.2(1.3,3.1)$ & $0.86(0.58,1.28)$ & $0.9(0.2,1.5)$ & $0.71(0.42,1.20)$ & $0.7(0.6,0.9)$ & $0.84(0.61,1.14)$ \\
\hline 5th quintile (high) & $1.9(1.7,2.1)$ & $1.02(0.73,1.43)$ & $2.3(1.2,3.5)$ & $0.73(0.48,1.13)$ & $1.0(0.5,1.6)$ & $0.83(0.48,1.43)$ & $0.9(0.7,1.1)$ & $1.02(0.73,1.43)$ \\
\hline ALL & $2.0(1.8,2.2)$ & - & $2.3(1.4,3.2)$ & - & $1.1(0.4,1.9)$ & - & $0.9(0.5,1.3)$ & - \\
\hline
\end{tabular}

rates for other subgroups of the maternity population $[11,38]$.

Hospital-level factor adjustment of planned caesarean section rates, lead to a much greater reduction in variation (by a further $31.7 \%$ ). Similar to other studies $[38,43]$, the odds of both, planned caesarean section and intrapartum caesarean section, are increased for private hospitals compared to public hospitals, suggesting that there are other factors driving the decision for caesarean section at these hospitals, irrespective of whether labour is attempted. It is unknown whether these factors are associated with women's or clinician's management preferences. Other hospital factors associated with increased odds of planned caesarean section were an overall larger proportion of low risk deliveries at the hospital and a lower hospital propensity towards vaginal birth after caesarean. These factors suggest that there are features or cultural aspects of certain hospitals that influence the likelihood that a patient who has had at least one caesarean section will receive a planned caesarean section. Many guidelines recommend that a vaginal birth after a caesarean section only be offered in a hospital that has available resources for an immediate caesarean section [44-46], and so a high planned caesarean section rate may be appropriate if hospitals have difficulty accessing theatres or anaesthetic staff to perform rapid emergency caesarean section following a trial of labour after a caesarean section $[47,48]$. Variation associated with hospital characteristics may be modifiable with hospital level interventions such as written guidelines for standardised management having been shown to be associated with an increase in the vaginal birth after caesarean section rate [49, 50]. Targeted hospital level interventions such as introduction of regular caesarean section audits and hospital funding tied to the hospital caesarean section rate have also been shown to increase vaginal birth after caesarean section rates with a concurrent downward trend in perinatal mortality and no change in maternal mortality [51]. There are currently no specific Australian guidelines for the management of multiparous women with at least one caesarean and a single cephalic pregnancy at term (Robson group 5). New South Wales state policy [52] recommends hospitals develop local guidelines, but the uptake, content and diversity of hospital-specific guidelines is unknown. Hospitals that used oxytocin among women with a previous caesarean section were more likely to achieve vaginal birth, consistent with previous work [53] and this factor may be modifiable, if appropriate.

Overall, only about half of the variation in hospital rates of planned caesarean section rates was explained by casemix and hospital-level factors, suggesting that other important factors are not captured in these data. Patients' and clinician's preferences vary and may explain the remaining variation [54]. Attitudes and practices of physicians have been previously shown to strongly influence patient preferences in their choice of repeat caesarean

Table 5 Case-mix adjusted morbidity rates across quintiles of hospital intrapartum caesarean section rates in NSW, 2007-2011

\begin{tabular}{|c|c|c|c|c|c|c|c|c|}
\hline \multirow[t]{2}{*}{ Hospital quintiles } & \multicolumn{2}{|c|}{ Maternal morbidity } & \multicolumn{2}{|c|}{ Neonatal morbidity } & \multicolumn{2}{|c|}{ Postpartum haemorrhage } & \multicolumn{2}{|c|}{ Apgar score at 5 minutes $<7$} \\
\hline & Rate $(95 \% \mathrm{Cl})$ & OR $(95 \% \mathrm{Cl})$ & Rate $(95 \% \mathrm{Cl})$ & OR $(95 \% \mathrm{Cl})$ & Rate $(95 \% \mathrm{Cl})$ & OR $(95 \% \mathrm{Cl})$ & Rate $(95 \% \mathrm{Cl})$ & OR $(95 \% \mathrm{Cl})$ \\
\hline \multicolumn{9}{|c|}{ Intrapartum caesarean } \\
\hline 1st quintile (low) & $2.8(2.4,3.2)$ & $0.95(0.61,1.49)$ & $2.7(2.0,3.3)$ & $0.65(0.43,0.99)$ & $9.0(7.6,10.4)$ & $0.73(0.55,0.97)$ & $1.5(1.3,1.7)$ & $0.96(0.70,1.32)$ \\
\hline 2nd quintile & $3.3(2.9,3.6)$ & $1.56(1.03,2.35)$ & $3.0(2.2,3.9)$ & $0.70(0.46,1.08)$ & $12.0(9.6,14.3)$ & $0.94(0.72,1.23)$ & $1.8(1.3,2.2)$ & $0.78(0.57,1.08)$ \\
\hline 3rd quintile & $2.7(2.6,2.9)$ & 1.00 & $3.6(2.3,4.9)$ & 1.00 & $11.4(9.0,13.8)$ & 1.00 & $1.5(1.3,1.7)$ & 1.00 \\
\hline 4th quintile & $2.8(2.5,3.1)$ & $0.15(0.70,1.88)$ & $2.5(1.8,3.1)$ & $0.55(0.34,0.88)$ & $9.4(7.7,11.0)$ & $0.72(0.54,0.98)$ & $1.5(1.4,1.6)$ & $0.84(0.61,1.14)$ \\
\hline 5th quintile (high) & $2.7(2.6,2.9)$ & $1.04(0.65,1.67)$ & $2.3(1.5,3.0)$ & $0.47(0.29,0.77)$ & $8.8(7.3,10.3)$ & $0.66(0.46,0.92)$ & $1.5(1.2,1.8)$ & $1.02(0.73,1.43)$ \\
\hline ALL & $2.9(2.5,3.3)$ & - & $2.8(1.9,3.8)$ & - & $10.3(7.9,12.6)$ & - & $1.6(1.3,1.9)$ & - \\
\hline
\end{tabular}


section or VBAC [55], and may be modifiable, through interventions such as an educational strategy delivered by an opinion leader [56]. The remaining variability may also, in part, reflect uncertainty among physicians on how to best balance the benefits and risk of repeat caesarean section in women who have had a previous caesarean section [54]. In addition to being strongly influenced by practitioners, women's preferences have been shown to be influenced by their individual experience and risk assessment, family commitments, safety concerns for the baby, their desire for predictability of birth and recovery, fear of labour or pain and a desire for sterilisation. [54]. An understanding of women's preferences and physician's decision-making is essential for the evaluation of quality and appropriateness of obstetric care provided to women [54] and warrants further investigation using a mixture of quantitative and qualitative approaches.

Rates of intrapartum caesarean section rates among women undergoing a trial of labour varied widely across hospitals. Following adjustment for case-mix, 16 of 73 hospitals had rates significantly different from the state average rate of intrapartum caesarean section following a trial of labour. This suggests that women with the same characteristics undergoing a trial of labour would have different risks of having an intrapartum caesarean section depending on the hospital attended. Not only is there variation between hospitals in being offered a trial of labour, the threshold to intervene once in labour appears to also differ. Hospital factors only explained a small proportion of the remaining variation. Notably, hospitals with high rates of instrumental delivery and VBAC for women after a caesarean for breech presentation had lower rates of intrapartum caesarean section. There is a trade-off with a higher instrumental delivery rate associated with lower odds of intrapartum caesarean section. These two factors may be modifiable and evidence for nulliparous women suggests that written guidelines for standardised labour management may provide an option to reduce the variation in intrapartum caesarean section rates between hospitals [50].

The appropriateness of caesarean section rates can be assessed by examination of the related morbidity and mortality. This study found higher rates of maternal and neonatal morbidity for a trial of labour compared to planned repeat caesarean section, consistent with previous work [57]. The observed higher rates of neonatal morbidity for an intrapartum caesarean section compared to a planned vaginal birth after caesarean are expected [57]. Similarly, the higher rates of postpartum haemorrhage at intrapartum compared to planned caesarean section are consistent with other studies [58]. Encouragingly, despite some increased variation for neonatal morbidity, lower rates of planned caesarean section were not associated with worse maternal or neonatal outcomes, suggesting that modification of rates should not have adverse effects. However, four outlier hospitals caused a triangular pattern of association between adjusted morbidity rates and quintiles of adjusted intrapartum caesarean section rates. Removal of these hospitals from analyses resulted in no significant associations, consistent with another study of women with previous caesarean section. That study found no differences between adjusted morbidity rates of obstetric residency program and non-residency program hospitals and rates of vaginal birth after caesarean section [48]. The reasons for the particularly high rates of morbidity for the outlier hospitals in the second and third intrapartum caesarean section quintiles are unknown and could be investigated via clinical audit. Hospitals identified in this study with low repeat caesarean section rates and low maternal and neonatal morbidities could also provide valuable insights for the improvement of maternity care.

\section{Conclusions}

Hospital rates of planned and intrapartum caesarean section for women with at least one previous caesarean section vary widely and only some of the variation can be explained by case-mix and hospital-level factors, suggesting that additional factors influence practices. Hospital rates of planned caesarean section are not associated with morbidity, yet morbidity rates differed among quintiles of hospital intrapartum caesarean section rates in a nonsystematic manner, influenced by a few outlying rates. Instituting hospital practice changes requires monitoring of morbidity to ensure no adverse effects.

\section{Competing interests}

The authors declare that they have no competing interests.

\section{Authors' contributions}

CLR and JBF conceived the study. All co-authors were involved in study design, interpretation of the results and reviewing the manuscript. KS prepared and analysed the data and drafted the manuscript. JAP provided statistical advice. All authors read and approved the final manuscript.

\section{Acknowledgements}

We thank the NSW Ministry of Health for access to the population health data and the Centre for Health Record Linkage ( $\mathrm{CHeReL}$ ) for linkage of the data sets. This work was completed while KS was employed as a trainee on the Biostatistics Training Program funded by the NSW Ministry of Health. She undertook this work whilst based at the Kolling Institute of Medical Research. JBF is supported by an Australian Research Council Future Fellowship (FT120100069); and CLR by an Australian National Health and Medical Research Council Senior Research Fellowship (1021028).

\section{Author details}

${ }^{1}$ Clinical and Population Perinatal Health Research, Kolling Institute of Medical Research, University of Sydney at Royal North Shore Hospital, St Leonards, NSW 2065, Australia. ${ }^{2}$ NSW Biostatistics Training Program, NSW Ministry of Health, North Sydney, NSW 2060, Australia. ${ }^{3}$ Department of Obstetrics and Gynaecology, Royal North Shore Hospital, Northern Sydney Local Health District, St Leonards, NSW 2065, Australia. 
Received: 6 May 2015 Accepted: 4 August 2015

\section{Published online: 19 August 2015}

\section{References}

1. AlHW. Australia's mothers and babies 2012. In: Perinatal statistics series no 30 Cat no PER 69. Canberra: Australian Institute of Health and Welfare (AlHW); 2014.

2. NHS Information Centre (NHS IC): NHS maternity statistics, England: 2013-14. In: Health and Social Care Information Centre, Hospital Episode Statistics. Leeds: [http://www.hscic.gov.uk/catalogue/ PUB16725/nhs-mate-eng-2013-14-summ-repo-rep.pdf] Accessed 19 March 2015; 2015.

3. Osterman M, Martin J: Changes in cesarean delivery rates by gestational age: United States, 1996-2011. In. Hyattsville, MD: National Center for Health Statistics; 2013.

4. Chu K, Cortier H, Maldonado F, Mashant T, Ford N, Trelles M. Cesarean Section Rates and Indications in Sub-Saharan Africa: A Multi-Country Study from Medecins sans Frontieres. PLoS ONE. 2012;7(9), e44484.

5. Ye J, Betrán A, Guerrero Vela M, Souza J, Zhang J. Searching for the optimal rate of medically necessary cesarean delivery. Birth. 2014;41(3):237-44.

6. World Health Organization. Appropriate technology for birth. Lancet. 1985:2:436-7.

7. World Health Organization: Monitoring obstetric care: a handbook. In. Geneva, Switzerland: WHO Press, World Health Organization; 2009.

8. Brennan D, Murphy M, Robson M, O'Herlihy C. The singleton, cephalic, nulliparous woman after 36 weeks of gestation - Contribution to the overall cesarean delivery rates. Obstetrics and Gynecology. 2011;117(2):273-9.

9. Roberts C, Algert C, Ford J, Todd A, Morris J. Pathways to a rising caesarean section rate: a population-based cohort study. BMJ Open. 2012;2, e001725.

10. Robson M. Classification of caesarean sections. Fetal Matern Med Rev. 2001;12:23-39.

11. Lee Y, Roberts C, Patterson J, Simpson J, Nicholl M, Morris J, et al. Unexplained variation in hospital caesarean section rates. Medical Journal of Australia. 2013;199:348-53.

12. Robson M, Hartigan L, Murphy M. Methods of achieving and maintaining an appropriate caesarean section rate. Best Practice \& research Clinical Obstetrics \& Gynaecology. 2013;27(2):297-308

13. Brennan D, Robson M, Murphy MH C. Comparative anlysis of international cesarean delivery rates using 10-group classification identifies significant variation in spontaneous labor. American Journal of Obstetrics and Gynecology. 2009:201:308. e301-308.

14. Betrán A, Vindevoghel N, Souza J, Gülmezoglu A, Torloni M. A systematic review of the Robson classification for caesarean section: What works, doesn't work and how to improve it. PLoS ONE. 2014;9(6), e97769.

15. Robson M. Can we reduce the caesarean section rate? Best Pract Res Clin Obstet Gynaecol. 2001;15:179-94.

16. Slavin V, Fenwick J. Use of a Classification Tool to Determine Groups of Women That Contribute to the Cesarean Section Rate: Establishing a Baseline for Clinical Decision Making and Quality Improvement. International Journal of Childbirth. 2012;2:85-94.

17. Farine D, Shepherd D. Classification of caesarean sections in Canada: the modified robson criteria. J Obstet Gynaecol Can. 2012;34:976-9.

18. Betran A, Gulmezoglu A, Robson M, Merialdi M, Souza J, et al. WHO global survey on maternal and perinatal health in Latin America: classifying caesarean sections. Reproductive Health. 2009;6:18.

19. Costa M, Cecatti J, Souza J, Milanez H, Gulmezoglu M. Using a Caesarean Section Classification System based on characteristics of the population as a way of monitoring obstetric practice. Reproductive Health. 2010;7:13.

20. Birkmeyer JD, Reames BN, McCulloch P. Understanding of regional variation in the use of surgery. Lancet. 2013;382:1121-9.

21. Synnes A, Macnab Y, Qiu Z, Ohlsson A, Gustafson P, Dean C, et al. Neonatal intensive care unit characteristics affect the incidenceof severe intraventricular hemorrhage. Med Care. 2006:44:754-9.

22. Lee S, McMillan D, Ohlsson A, Pendray M, Synnes A, Whyte R, et al. Variations in practice and outcomes in the Canadian NICU network: 1996-1997. Pediatrics. 2000;106:1070-9.

23. Australian Bureau of Statistics: Australian Demographic Statistics. In. Canberra: Australian Bureau of Statistics; 2011.

24. National Centre for Classification in Health ( $\mathrm{NCCH})$ : The International Statistical Classification of Diseases and Related Health Problems, Tenth Revision, Australian Modification (ICD-10-AM), Australian Classification of
Health Interventions (ACHI) and Australian Coding Standards (ACS). In., 7th Edition edn. Sydney: University of Sydney; 2010.

25. Bentley J, Ford J, Taylor L, Irvine K, Roberts C. Investigating linkage rates among probabilistically linked birth and hospitalization records. BMC Medical Research Methodology. 2012;12:149

26. Roberts C, Nicholl M, Algert C, Ford J, Morris J, Chen J. Rate of spontaneous onset of labour before planned repeat caesarean section at term. BMC Pregnancy and Childbirth. 2014;14:125.

27. Census of Population and Housing: Socio-Economic Indexes for Areas (SEIFA) [http://www.abs.gov.au/websitedbs/censushome.nsf/home/ seifa?opendocument\&navpos=260]

28. Centre for Epidemiology and Evidence: Health Statistics New South Wales. In. Sydney: NSW Ministry of Health; 2014.

29. Falster M, Roberts C, Ford J, Morris J, Kinnear A, Nicholl M. Development of a maternity hospital classification for use in perinatal research. NSW Public Health Bulletin. 2012;23(2):12-6.

30. Taylor L, Bajuk B, Sutton L, Travis S, Banks C. Validation study: NSW Midwives Data Collection 1998. NSW Public Health Bulletin Supplementary Series. 2000;11:97-99.

31. Roberts $C B$ JC, Ford J, Morris J. Monitoring the quality of maternity care: how well are labour and delivery events reported in population health data? Paediatric and Perinatal Epidemiology. 2009;23(2):144-52.

32. Roberts C, Bell J, Ford J, Hadfield R, Algert C, Morris J. The accuracy of reporting of the hypertensive disorders of pregnancy in population health data. Hypertension in Pregnancy. 2008;27(3):285-97.

33. Roberts C, Lain S, Hadfield R. Quality of population health data reporting by mode of delivery. Birth. 2007;34(3):274-5.

34. Taylor L, Travis S, Pym M, Olive E, Henderson-Smart D. How useful are hospital morbidity data for monitoring conditions occurring in the perinatal period? Australian and New Zealand Journal of Obstetrics and Gynaecology. 2005:45:36-41.

35. Roberts C, Cameron C, Bell J, CS A, Morris J. Measuring maternal morbidity in routinely collected health data: development and validation of a maternal morbidity outcome indicator. Med Care. 2008;46:786-94.

36. Henderson T, Shepheard J, Sundararajan V. Quality of diagnosis and procedure coding in ICD-10 administrative data. Medical Care. 2006:44(11):1011-9.

37. Hadfield R, Lain S, Cameron C, Bell J, Morris J, Roberts C. The prevalence of maternal medical conditions during pregnancy and a validation of their reporting in hospital discharge data. Australian and New Zealand Journal of Obstetrics and Gynaecology. 2008;48:78-82.

38. Nippita T, Lee Y, Patterson J, Ford J, Morris J, Nicholl M, et al. Variation in hospital caesarean section rates and obstetric outcomes among nulliparae at term: a population-based cohort study. BJOG. 2015. doi:10.1111/14710528.13281.

39. Lain S, Algert C, Nassar N, Bowen J, Roberts C. Incidence of severe adverse neonatal outcomes: use of a composite indicator in a population cohort. Matern child Health J. 2012:16:600-8.

40. Lain S, Roberts C, Hadfield R, Bell J, Morris J. How accurate is the reporting of obstetric haemorrhage in hospital discharge data? Aust NZ J Obstet Gynaecol. 2008;48:481-4.

41. Algert C, Morris J, Simpson J, Ford J, Roberts C. Labor before a primary cesarean delivery: reduced risk of uterine rupture in a subsequent trial of labour for vaginal birth after cesarean. Obstetrics and Gynecology. 2008;112(5):1061-6.

42. Gross M, Matterne A, Berlage S, Kaiser A, Lack N, Macher-Heidrich S, et al. Interinstitutional variations in mode of birth after a previous caesarean section: a cross-sectional study in six German hospitals. Journal of Perinatal Medicine. 2015. doi:10.1515/jpm-2014-0108.

43. Murphy D, Fahey T. A retrospective cohort study of mode of delivery among public and private patients in an integrated maternity hospital setting. BMJ Open. 2013;3, e003865

44. The Royal Australian and NewZealand College of Obstetricians and Gynaecologists: Planned vaginal birth after caesarean section (trial of labour). In: C-Obs 38. The Royal Australian and NewZealand College of Obstetricians and Gynaecologists; 2010.

45. RCOG: Birth after previous caesarean birth. In: Green-top Guideline No 45. Accessed at https://www.rcog.org.uk/globalassets/documents/ guidelines/gtg4511022011.pdf: Royal College of Obstetricians and Gynaecologists (RCOG); 2007.

46. ACOG: Vaginal birth after previous cesarean delivery. In: Practice Bulletin Number 115. The American College of Obstetricians and Gynecologists (ACOG); 2010 
47. Scott J. Intrapartum management of a trial of labour after caesarean delivery: evidence and experience. An International Journal of Obstetrics and Gynaecology. 2013;121:157-62.

48. DeFranco E, Rampersad R, Atkins K, Odibo A, Stevens E, Peipert J, et al. Do vaginal birth after cesarean outcomes differ based on hospital setting? American Journal of Obstetrics and Gynecology. 2007;197:400. e401-400.e406.

49. Gardner K, Henry A, Thou S, Davis G, Miller T. Improving VBAC rates: the combined impact of two management strategies. Aust N Z J Obstet Gynaecol. 2014;54(4):327-32.

50. Alfirevic Z, Edwards G, Platt M. The impact of delivery suite guidelines on intra-partum care in 'standard primigravida'. European Journal of Obstetrics \& Gynecology and Reproductive Biology. 2004;115:28-31.

51. Ayres-De-Campos D, Cruz J, Medeiros-Borges C, Costa-Santos C, Vicente L. Lowered national cesarean section rates after a concerted action. Acta Obstet Gynecol Scand. 2015;94(4):391-8.

52. NSW Department of Health: Maternity - Towards normal birth in NSW. In. Sydney: NSW Department of Health; 2010

53. Roberts C, Algert C, Nippita T, Morris J. Trial of labour after cesarean (TOLAC): outcomes related to obstetric history and labor at TOLAC. American Journal of Obstetrics and Gynecology. 2015;212(1, Supplement):S309-10.

54. Kaimal A, Kuppermann M. Understanding risk, patient and provider preferences, and obstetrical decision making: Approach to delivery after cesarean. Seminars in Perinatology. 2010;34(5):331-6.

55. Bernstein SN, Matalon-Grazi S, Rosenn BM. Trial of labor versus repeat cesarean: are patients making an informed decision? Am J Obstet Gynecol. 2012;207(3):204. e201-206.

56. Lundgren I, Smith V, Nilsson C, Vehvilainen-Julkunen K, Nicoletti J, Devane $D$, et al. Clinician-centred interventions to increase vaginal birth after caesarean section (VBAC): a systematic review. BMC Pregnancy Childbirth. 2015;15(1):16.

57. Hook B, Kiwi R, Amini S, Fanaroff A, Hack M. Neonatal morbidity after elective repeat caesarean section and trial of labor. Pediatrics. 1997;100(3 Pt 1):348-53.

58. Ford J, Roberts C, Simpson J, Vaughan J, Cameron C. Increased postpartum haemorrhage rates in Australia. International Journal of Gynaecology and Obstetrics. 2007;98(3):237-43.

\section{Submit your next manuscript to BioMed Central and take full advantage of:}

- Convenient online submission

- Thorough peer review

- No space constraints or color figure charges

- Immediate publication on acceptance

- Inclusion in PubMed, CAS, Scopus and Google Scholar

- Research which is freely available for redistribution 